\title{
Addressing the challenge to identify the role played by lifestyle factors in dementia
}

\author{
Tasnime Akbaraly ${ }^{1,2}$ \\ ${ }^{1}$ Université Paris-Saclay, UVSQ, Inserm, CESP, "DevPsy”, Villejuif, France; ${ }^{2}$ Department of Epidemiology and Public Health, University College \\ London, London, UK \\ Correspondence to: Tasnime Akbaraly. INSERM U1018, Hôpital Paul Brousse, 16 avenue Paul Vaillant Couturier, 94807 Villejuif, France. \\ Email: tasnime.akbaraly@inserm.fr. \\ Provenance and Peer Review: This article was commissioned and reviewed by the Academic Editor Dr. Zuo-Teng Wang (Department of Neurology, \\ Qingdao Municipal Hospital, College of Medicine and Pharmaceutics, Ocean University of China, Qingdao, China). \\ Comment on: Lourida I, Hannon E, Littlejohns TJ, et al. Association of Lifestyle and Genetic Risk With Incidence of Dementia. JAMA 2019. [Epub \\ ahead of print].
}

Submitted Dec 20, 2019. Accepted for publication Jan 13, 2020.

doi: $10.21037 / \mathrm{atm} .2020 .01 .74$

View this article at: http://dx.doi.org/10.21037/atm.2020.01.74

In a context of absence of curative treatments for dementia, strategies consisting in identifying modifiable risk factors in order to delay cognitive decline and prevent onset of dementia remain crucial. The strong association between cardiovascular risk and all-cause dementia fostered an extensive observational cohort research-based examining the extent to which lifestyle factors predicting cardiometabolic disorders were also predicting cognitive disorders and dementia.

In parallel, beyond Apolipoprotein E genotype-the major genetic risk factor for late-onset, "sporadic" forms of $\mathrm{AD}$ (LOAD), findings from 10-year research based on genome-wide association studies allows the identification of common genetic variations at over 20 loci associated with LOAD (outside of the APOE locus).

The present editorial will comment the paper led by Lourida et al. recently published in $7 A M A$ (1), in which, by adopting an integrative approach, authors assessed whether a healthy lifestyle was associated with lower risk of dementia regardless of genetic risk using the large UK Biobank cohort. Authors were able to build a polygenic risk score-capturing the individual's load of common genetic variants associated with $\mathrm{AD}$ and dementia - and to combine four health behaviors associated with dementia (smoking, diet, physical activity and alcohol consumption) to compute a "healthy lifestyle" score. Quantitative analyses of the interplay between genetic and lifestyle factors regarding dementia risk have been conducted in 200,000 participants, aged 60 years and over for whom dementia diagnosis $(1,769$ cases) was obtained through linkage to electronical records over a median follow-up period of 8 years. Findings showed that both multiple genetic risk factors and combination of lifestyle factors were significantly and independently associated with dementia risk. Participants at high genetic risk and with unfavorable lifestyles showed a nearly threefold increased risk of dementia [hazard ratio (HR): 2.83; 95\% confidence interval (CI), 2.09-3.83] compared to participants with low genetic risk and favorable lifestyles. With a significant dementia risk reduction in participants with favorable lifestyles compared to those with unfavorable lifestyles whatever the genetic risk, the findings suggest that improving health behaviors can be a potential lever on which intervention strategies can be operated to prevent dementia onset. Actually, based on the estimates in their UK Biobank sample, authors make the hypothesis that even in high genetic risk population some dementia cases might be prevented ( 1 on 121 per 10 years) if individuals improved their lifestyle from unfavorable to favorable categories.

This combined analysis of health behavior across genetic risk has been applied first to cardiovascular diseases and type 2 diabetes via two studies $(2,3)$. The first one, conducted by Khera et al., was carried out on three prospective cohorts (the Atherosclerosis Risk in Communities, Women's Genome Health Study and Malmö Diet and Cancer Study) and the 


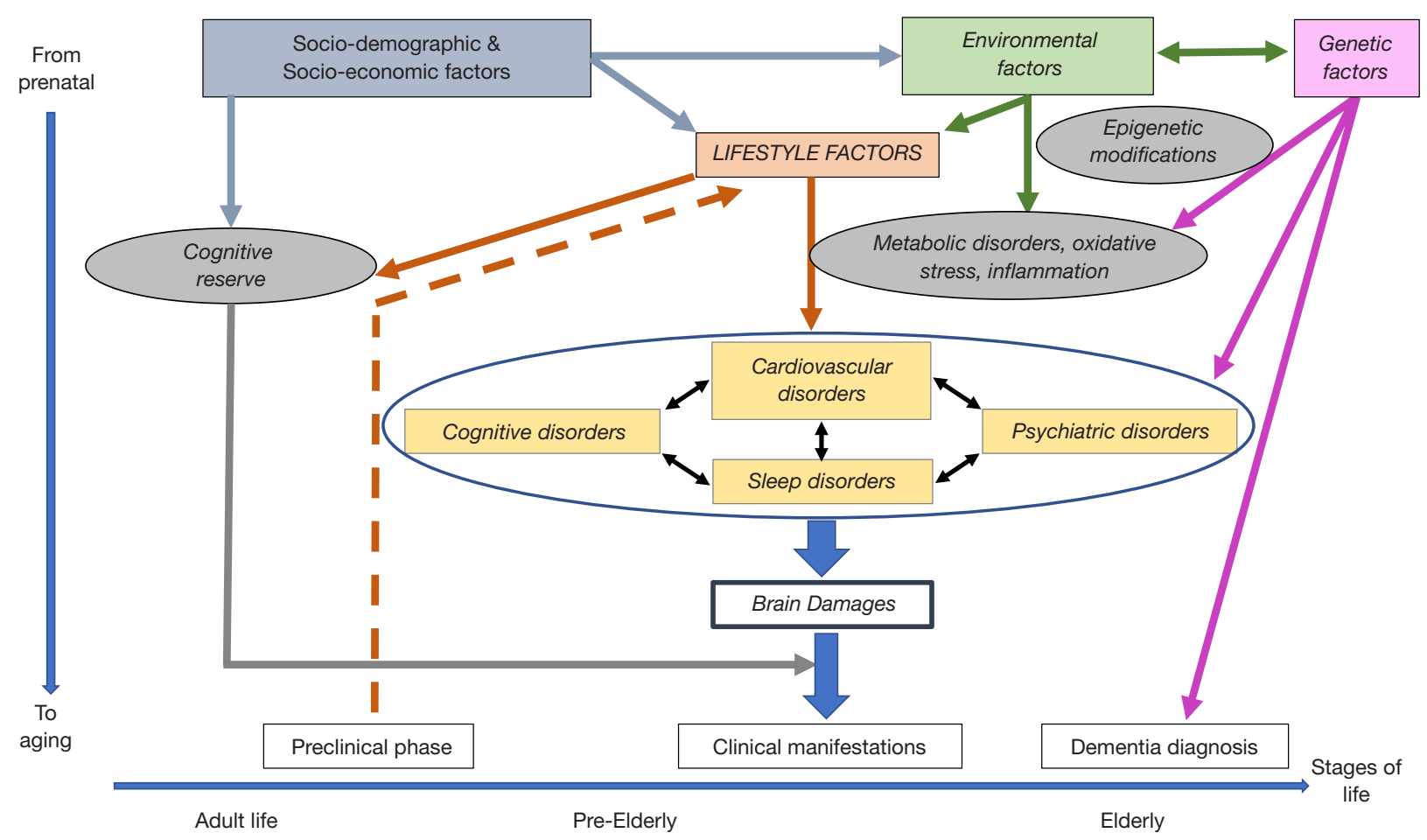

Figure 1 Complexity underlying the identification of the role played by lifestyle factors in dementia: risk factor or prodromal factor? Genetic, social, health behavior, environmental and health status factors associated with dementia are interrelated, and their association with dementia may change across the stages of life. Several hypotheses regarding the role of lifestyle factors on dementia onset co-exist. First, unhealthy lifestyle factors are hypothesized to have a causal role in dementia by inducing metabolic and biological dysregulations involved in development of diseases leading to dementia. Second, based on the concept of brain/cognitive reserve, healthy lifestyle might afford protection to dementia by reinforcing brain structures and developing the efficiency of neural network and cognitive processes underlying task performance to allow some people to cope better than others with brain damages that occur in dementia. Third, unfavorable lifestyle factors are dementia prodromal factors. Dementia is characterized by a long preclinical period (estimated between 15 to 20 years), involving pathophysiological changes. This period is characterized by an accelerated cognitive decline, changes in mood and depression that are likely to affect lifestyles.

second one was based on a US Biobank cohort. Findings from both reports indicate the strong potential benefits of adherence to multiple ideal lifestyle behaviors including (nonsmoking, healthy diet, healthy weight and regular physical activity) regardless genetic risk. Analyses from the Biobank study showed that over the 6-year of followup $37 \%(95 \%$ CI, 33-41) and $72 \%(95 \%$ CI, 68-76) of new onset of coronary arterial diseases and type 2 diabetes respectively might have been prevented if all individuals adhered to an ideal lifestyle behavior. Lessons from these two studies clearly showed that the combined analyses of genetic and lifestyle risk factors are worthwhile to further provide preventive policies for CVD and type 2 diabetes. However, more caution is required regarding interpretation when these combined analyses are applied to dementia.

By contrast to cardiometabolic disorders (where the causal effect of all four health behaviors have been established), no consistent conclusions can be drawn and the role of lifestyle factors in dementia onset. This is mainly due to the conflicting results from prospective cohort studies and the inaccuracies on the period of life during which favorable lifestyles might afford protection against dementia. The understanding of health behaviordementia association in observational studies has to take into account the preclinical period preceding the diagnosis, the phenotypic heterogeneity of dementia and the complex interplay of genetic, socio-economic status, lifestyle and health status (cardiometabolic, depressive, sleep disorders), as illustrated in Figure 1.

Indeed, dementia is characterized by a long preclinical period, involving pathophysiological changes over several years. This period, estimated between 15 to 20 years, is 
notably characterized by an accelerated cognitive decline, changes in mood and depression that are likely to affect lifestyles. Thus, prospective studies with under 15-year follow-ups do not allow to understand the role played by healthy behaviour-a modifiable protective factor or a prodromal factor or both according to the considered lifetime window?

This can be illustrated by findings from a collaborative study led by Kivimaki et al. showing in 1.3 million adults that a higher BMI was associated with increased risk of dementia when weight was measured more than 20 years before dementia diagnosis but this association was reversed when BMI was assessed less than 10 years before (4). Authors conclude that the BMI-dementia association described is likely to be attributable to two processes: a direct harmful effect of higher BMI and a reverse causation effect as a result of weight loss during the preclinical dementia phase.

Regarding diet, studies carried out in the Atherosclerosis Risk in Communities (5) and Whitehall II (6) cohorts did not report associations between diet quality assessed at midlife and new onset of dementia after follow-ups longer than 20 years, while meta-analyses of prospective studies with follow-ups shorter than 10 years showed that low diet is associated with high dementia risk. Taking together these findings might actually suggest a prodromal role of diet rather than a role of protective factor. Further studies are needed to examine underlying mechanisms for the deterioration of lifestyle behaviours during the preclinical stage of the diseases. However, other explanations than reversion causation effect of diet can be proposed. Given the causal effect of diet on CVD and the strong vascular component in neurodegenerative dementia, the likelihood that diet would exert a protective effect only in specific dementia subgroups is also probable. Actually, dementia can result from a number of complex disorders which damage the brain, the heterogeneity of its clinical manifestation as well as the absence of clear biomarkers make the dementia subgroups diagnosis very challenging especially within large observational cohort frameworks where dementia diagnosis was obtained through electronic data linkage.

Further research is needed to examine whether the role of genetic, lifestyle, and more widely environmental factors vary according to dementia phenotypes.

\section{Acknowledgments}

Funding: None.

\section{Footnote}

Conflicts of Interest: The author has completed the ICMJE uniform disclosure form (available at http://dx.doi. org/10.21037/atm.2020.01.74). The author has no conflicts of interest to declare.

Ethical Statement: The author is accountable for all aspects of the work in ensuring that questions related to the accuracy or integrity of any part of the work are appropriately investigated and resolved.

Open Access Statement: This is an Open Access article distributed in accordance with the Creative Commons Attribution-NonCommercial-NoDerivs 4.0 International License (CC BY-NC-ND 4.0), which permits the noncommercial replication and distribution of the article with the strict proviso that no changes or edits are made and the original work is properly cited (including links to both the formal publication through the relevant DOI and the license). See: https://creativecommons.org/licenses/by-nc-nd/4.0/.

\section{References}

1. Lourida I, Hannon E, Littlejohns TJ, et al. Association of Lifestyle and Genetic Risk With Incidence of Dementia. JAMA 2019. [Epub ahead of print].

2. Khera AV, Emdin CA, Drake I, et al. Genetic Risk, Adherence to a Healthy Lifestyle, and Coronary Disease. N Engl J Med 2016;375:2349-58.

3. Said MA, Verweij N, van der Harst P. Associations of Combined Genetic and Lifestyle Risks With Incident Cardiovascular Disease and Diabetes in the UK Biobank Study. JAMA Cardiol 2018;3:693-702.

4. Kivimäki M, Luukkonen R, Batty GD, et al. Body mass index and risk of dementia: Analysis of individual-level data from 1.3 million individuals. Alzheimers Dement 2018;14:601-9.

5. Dearborn-Tomazos JL, Wu A, Steffen LM, et al. Association of Dietary Patterns in Midlife and Cognitive Function in Later Life in US Adults Without Dementia. JAMA Netw Open 2019;2:e1916641.

6. Akbaraly TN, Singh-Manoux A, Dugravot A, et al. Association of Midlife Diet With Subsequent Risk for Dementia. JAMA 2019;321:957-68.

Cite this article as: Akbaraly T. Addressing the challenge to identify the role played by lifestyle factors in dementia. Ann Transl Med 2020;8(11):724. doi: 10.21037/atm.2020.01.74 